\title{
Ambiente escolar y su importancia en la calidad educativa: una perspectiva neuropedagógica
}

\section{School environment and its importance in educational quality: a neuropedagogical approach}

Sweney Oriana Giraldo-Aristizabal (D)

Universidad del Atlántico, Barranquilla, Colombia

Marleyn Margarita Serrano-Ramírez (D)

Universidad del Atlántico, Barranquilla, Colombia

\section{Resumen}

Objetivo: Identificar las percepciones que tienen los estudiantes de sus docentes con relación al manejo de las habilidades emocionales y comunicativas y el ambiente escolar en una institución educativa privada. Método: Se desarrolló un enfoque de métodos mixtos con un diseño explicativo secuencial a través de la combinación de técnicas cuantitativas (Cuestionario, test y lista de chequeo) y cualitativas (grupos focales) aplicados a 18 docentes y 131 estudiantes de básica secundaria y media vocacional de la institución educativa privada. Resultados: A través de esta investigación se identificó que la percepción de los estudiantes difiere con la de los docentes en cuanto al manejo de las habilidades emocionales y comunicativas; los docentes no son conscientes de esta falencia. Discusión y conclusiones: El hallazgo afecta el ambiente escolar, por esto, se requiere una reflexión neuropedagógica de tal forma que el maestro sea consciente de la necesidad de comprender la trascendencia de la neuropedagogía en el proceso de enseñanza y aprendizaje, la importancia de la motivación y emoción en el aprendizaje de los estudiantes y optimizar la relación docente-estudiante.

Palabras clave: Ambiente escolar, habilidades emocionales, habilidades comunicativas, neuropedagogía.

\begin{abstract}
Objective: Identify the perceptions that students have of their teachers in relation to the management of emotional and communication skills and the school environment in a private educational institution. Method: A mixed methods approach with sequential explanatory design was developed through the use of the combination of quantitative techniques (questionnaire, test and check list) and qualitative techniques (focus groups) applied to 18 teachers and 131 students of Middle and High School levels of the private educational institution. Results: Throughout this investigation, it was identified that the perception of the students differs from that of the teachers concerning the management of emotional and communicational skills; the teachers are not aware of such misinterpretation. Discussion and conclusion: The finding affects the school environment, therefore, a neropedagogical reflection is required so that the teacher be aware of such need to understand the transcendence of the neuropedagogy in the teaching learning process, the importance of motivation and emotion in the students's learning process and optimizing the teacher-student relationship.
\end{abstract}

Keywords: School environment, emotional skills, communicational skills, neuropedagogy.
Open Access:

ISSN: $0124-2121$

E-ISSN: 2665-2420

$$
\begin{gathered}
\text { ARTÍCULO DE INVESTIGACIÓN } \\
\text { CIENTÍFICA } \\
\text { Copyright @ } \\
\text { By Educación y Humanismo } \\
\text { Editor: } \\
\text { Dhayana Fernández Matos } \\
\text { Universidad Simón Bolívar } \\
\text { Correspondencia: } \\
\text { Sweney Giraldo } \\
\text { sweneyoriana@gmail.com }
\end{gathered}
$$

Recibido: 28-05-2020 Aceptado: 03-09-2020 En línea desde: $14-12-2020$ 


\section{Introducción}

La educación en Colombia se rige por lineamientos, estándares, leyes, decretos, programas, entre otros, con el fin de propender por una educación de calidad, la cual es definida por el Ministerio de Educación Nacional (MEN, 2011) como:

Aquella que forma mejores seres humanos, ciudadanos con valores éticos, respetuosos de lo público, que ejercen los derechos humanos y conviven en paz. Una educación que genera oportunidades legítimas de progreso y prosperidad para ellos y para el país (...) Una educación competitiva, que contribuye a cerrar brechas de inequidad, centrada en la institución educativa y en la que participa toda la sociedad (p. 4)

Es por ello que, para lograr una alta calidad educativa en el país, el MEN creó el Índice Sintético de Calidad Educativa (ISCE), definido como el "instrumento de medición de la calidad educativa de los establecimientos educativos y de las entidades territoriales certificadas en educación"(Decreto 501 de 2016, Artículo 2.3.8.8.2.3.1, inciso 1). Cabe destacar que el ISCE incluye cuatro componentes: Progreso, Desempeño, Eficiencia y Ambiente Escolar (MEN, 2014).

Además, De Zubiría (2018) menciona que

(...) impactar la calidad tiene que ver con el clima de aula y el clima institucional (...) el clima del aula es la variable más asociada a la calidad de la educación. Esta variable influye más que todas las demás variables sumadas. De allí que hay que prestarle especial cuidado. Tenemos que garantizar que la escuela se comprometa con una formación más tolerante, más respetuosa de la diferencia y que sea un espacio para cultivar la empatía y la comprensión de los otros (párr. 14)

Es así como el clima escolar es un aspecto fundamental para lograr la calidad de la educación, se requiere que la formación de los estudiantes esté mediada por relaciones tolerantes y respetuosas, de esta forma, la relación docente-estudiante y estudiante-estudiante sea favorable y contribuya a los procesos de enseñanza y aprendizaje.

\section{Según Meiss (2017)}

La calidad es el eje de la educación (...) La definición extensa de calidad describe las características deseables de: los estudiantes (sanos y motivados) los procesos (docentes competentes que utilizan pedagogías activas,) los contenidos (programas de estudios relevantes), y los sistemas (buena administración y distribución de recursos equitativa, entornos educativos seguros, sanos e integrados, a fin de favorecer un excelente aprendizaje y niveles bien definidos de rendimiento para todos) (párr. 6)

A partir de lo anterior, para lograr la calidad en la educación se requiere que los actores del proceso educativo se comprometan a alcanzar las metas propuestas, que el proceso de enseñanza y aprendizaje esté mediado por estrategias pedagógicas innovadoras, motivación 
por parte de los estudiantes, planes de estudio con contenidos intencionados, también, debe primar la equidad en la distribución de los recursos.

Por su parte, el MEN (2015) afirma que:

El Ambiente Escolar puede ser entendido como el conjunto de relaciones entre los miembros de una comunidad educativa que se encuentra determinado por factores estructurales, personales y funcionales confiriendo a la institución educativa un modo de vivir y de establecer relaciones entre todos los actores que en ella conviven. Un ambiente escolar favorable también es fundamental para que los docentes se sientan seguros en la escuela, se disminuyan las probabilidades de que se desarrollen enfermedades generadas por el estrés y la angustia, y más importante aún, los niveles de compromiso con el aprendizaje de los estudiantes tienden a elevarse. Es así como se puede señalar que un ambiente positivo no sólo favorece los aprendizajes de los estudiantes, sino que contribuye a la consolidación de metas comunes definidas institucionalmente (p. 20)

Es así como el componente de Ambiente Escolar corresponde a la evaluación de las condiciones propicias para el aprendizaje en el aula de clase basándose en dos aspectos: Ambientes propicios para el aprendizaje en el aula (clima escolar) y seguimiento al aprendizaje (retroalimentación) (MEN, 2014). Este componente en la básica secundaria "equivaldrá al diez por ciento (10\%) del ISCE y se calculará de acuerdo con el puntaje promedio que obtengan los establecimientos educativos en las Encuestas de Factores Asociados que hacen parte de las pruebas Saber 5 y 9" (Decreto 501 de 2016, Artículo 2.3.8.8.2.3.1, inciso 1.1.4).

Es importante mencionar que la institución educativa privada de la ciudad de Barranquilla objeto de estudio de esta investigación ha obtenido los siguientes puntajes en el componente de Ambiente Escolar, los cuales fueron socializados en el Día de la Excelencia Educativa (Día E) del año 2018, siendo 1 el puntaje más alto que se puede obtener:

Tabla 1.

Resultados ISCE 2015 al 2018

\begin{tabular}{cccc}
\hline \multicolumn{2}{c}{$\mathbf{5}^{\circ}$ grado } & \multicolumn{2}{c}{$\mathbf{9}^{\circ}$ grado } \\
\hline Año & Puntaje & Año & Puntaje \\
\hline 2015 & 0,77 & 2015 & 0,74 \\
\hline 2016 & 0,76 & 2016 & 0,76 \\
\hline 2017 & 0,75 & 2017 & 0,77 \\
\hline 2018 & 0,77 & 2018 & 0,76 \\
\hline
\end{tabular}

Fuente: Elaboración propia

Cabe destacar que, en el desarrollo del Día E de los estudiantes, los voceros de cada grado ( $3^{\circ}$ a $11^{\circ}$ grado) manifestaron las siguientes percepciones sobre ciertos aspectos que requieren mejorar en la institución educativa: Motivar a los estudiantes resaltando y teniendo en cuenta sus talentos y habilidades, mejorar la retroalimentación a los trabajos y formas de evaluación, las didácticas de las clases y que éstas sean más lúdicas. 
Con base en lo anterior, un factor importante para tener en cuenta es la motivación de los estudiantes, la relación e interacción docente-estudiante y las estrategias de enseñanza y aprendizaje que implementan los docentes con el fin de favorecer los procesos que se desarrollan en la institución educativa. Es por esto que es necesario reflexionar sobre el componente de Ambiente Escolar y las necesidades de este. Así como también los docentes deben poseer competencias emocionales y comunicativas que le permitan enriquecer su quehacer pedagógico en pro de mantener una relación docente-estudiante óptima y de esta forma mejorar sus prácticas pedagógicas. Por lo cual, "La competencia emocional de los docentes es necesaria para su propio bienestar personal y para su efectividad y calidad a la hora de llevar a cabo los procesos de enseñanza-aprendizaje del aula, en general, y del desarrollo socioemocional en los alumnos" (Sutton y Wheatly, citado por Palomera, Fernández, Brackett, 2008, p. 440).

Por lo tanto, se destaca el concepto de las habilidades emocionales asociado a la inteligencia emocional definida como "la habilidad para percibir, asimilar, comprender y regular las propias emociones y la de los demás promoviendo un crecimiento emocional e intelectual" (Mayer y Salovey, 1997, p.10). De igual forma, Ortiz (2015) afirma que "las estrategias pedagógicas curriculares y evaluativas deben estar encaminadas en establecer las configuraciones cerebrales y deben contribuir a estimular la creación de nuevas redes y circuitos de comunicación neuronal, que permitan orientar la formación de los estudiantes basándonos en los avances de las neurociencias" (p. 28).

Es así como diversas investigaciones y estudios científicos respaldan la importancia de las habilidades emocionales en el contexto escolar. Es de resaltar la investigación realizada por Cejudo \& López (2017) quienes mencionan que los maestros identificaron la importancia y necesidad del dominio de la inteligencia emocional para el óptimo desempeño como docentes. Asimismo, López \& Oriol (2016) plantean que la competencia emocional ejerce una influencia mediadora entre el clima de aula y el rendimiento académico. Además, afirma Esquivel (2018) que:

La función del docente es que sus alumnos se sientan a gusto y puedan aprender los contenidos que se requieren, por lo tanto, debe crear un buen ambiente para el aprendizaje tomando en cuenta el lado humano de sus alumnos y partir de ello para que su aprendizaje sea más significativo (p. 11)

La investigación de Morton (2014), por su parte, proporciona una comprensión del impacto de la inteligencia emocional docente en el clima escolar. Los resultados indicaron que la inteligencia emocional del maestro puede desarrollarse o mejorarse y que afecta su percepción del clima escolar. Fernández (2019) en su investigación presenta la importancia de las emociones en el proceso de enseñanza aprendizaje, expresa que las emociones son parte de las competencias educativas en nuestros días. Además, Jennings y Greenberg, Sutton y Wheatly (citados por Pertegal, Castejón y Martínez, 2011) identificaron a través de su investigación la 
estrecha relación entre las competencias sociales y emocionales de los profesores y la efectividad y la calidad a la hora de llevar a cabo procesos de enseñanza-aprendizaje del aula.

Es importante mencionar que los docentes deben crear en el aula condiciones emocionales necesarias para el aprendizaje, el maestro que posea inteligencia emocional ayudará a construir aulas emocionalmente dispuestas para el aprendizaje significativo en sus alumnos; si por el contrario, el docente tiene un mal manejo de sus estados emocionales, puede provocar que sus alumnos se sientan amenazados, lo cual conlleva a que dejen de aprender, no se cree un ambiente de confianza y apertura emocional para tratar los conflictos propios del aula (Vásquez, 2008).

En esta perspectiva es relevante mencionar otra de las habilidades necesarias para optimizar la relación docente-estudiante: Las habilidades comunicativas, que comprenden "un proceso de interacción social, más o menos complejo, mediante el cual nos definimos mientras compartimos mensajes con otras personas que intervienen utilizando códigos de interpretación similares (...) la comunicación es un proceso fundamental de toda relación social" (Reyes y Garrido, 2012, p. 24), pues gracias a ella se regula la interacción de las personas haciéndola posible.

Dentro de las habilidades comunicativas se encuentran las habilidades sociales, que son "un conjunto de pensamientos, emociones y conductas que permiten relacionarse y convivir con otras personas de forma satisfactoria y eficaz" (Monjas, 2013, p. 83). Se sabe, por otra parte, que "la intervención psicopedagógica dirigida a cambiar o mejorar el clima social del centro y del aula y a fomentar relaciones interpersonales cordiales, son un elemento importante de prevención de conductas violentas y de problemas de disciplina en los centros" (Monjas, 2013, p. 85). Es así como:

El buen clima se explica por las buenas relaciones entre los miembros de la comunidad escolar, destacándose características como la cordialidad, la camaradería, la sonrisa, el cariño, la confianza y la solidaridad entre los compañeros. Es importante fomentar un medio de trabajo entusiasta, agradable y desafiante para los estudiantes y los profesores, poniendo énfasis en el respeto mutuo, la confianza, la tolerancia y el trabajo en equipo (Thorne, Centeno, Wetzell, 2009, p. 66)

Con base en lo anterior, la relación docente-estudiante estará mediada por la calidad de las habilidades sociales de los docentes con el fin de crear un ambiente óptimo que genere confianza y empatía entre ambos actores, lo que resulta de gran importancia en el proceso de enseñanza y aprendizaje. Esto se evidencia en la investigación realizada por Arias (2018) quien analizó el papel del maestro en la construcción de un ambiente escolar óptimo a partir de la interacción y comunicación y de esta forma mejorar la convivencia en la institución educativa. También, Gutiérrez \& Buitrago (2019) realizaron una revisión teórica que analiza cómo las habilidades socioemocionales aportan a ambientes de paz en la escuela basado en el clima escolar y las relaciones interpersonales de docentes y estudiantes. Además, Aristulle \& Paoloni 
(2019) en su estudio describieron y analizaron el rol de las emociones y de las habilidades socioemocionales en el proceso de conformación de una comunidad educativa. Asimismo, Ramos \& López (2020) realizaron su investigación basados en el interés de las pautas comunicativas de los profesores hacia los estudiantes en el salón de clases, mediadas por el contenido escolar y los recursos didácticos utilizados para favorecer el aprendizaje disciplinar.

Por su parte Brackett, Alster, Wolfe, Katulak, Fale, Brackett, Caruso, Mayer, Roberts, Barsade (citados por Cabello, Ruiz y Fernández, 2010) mencionan que:

La capacidad para identificar, comprender y regular las emociones es fundamental en el profesorado, ya que estas habilidades van a influir en los procesos de aprendizaje, en la salud física, en la calidad de las relaciones interpersonales y en el rendimiento académico y laboral (p. 44)

De igual forma, Casassús y Hargreaves citados por Cejudo \& López (2017) mencionan que:

(...) el rol docente implica una importante carga de trabajo emocional, tanto por lo que exige de sensibilidad a las emociones ajenas como por lo que exige de manejar apropiadamente las emociones propias y ajenas para facilitar y optimizar la calidad de las relaciones interpersonales que caracterizan a las organizaciones escolares (p. 30)

Además, Sutton y Wheatley citados por Cejudo \& López (2017) mencionan que "el incremento de las emociones positivas puede facilitar la creación de un clima de clase que favorezca el aprendizaje" (p. 30).

Tomando en cuenta lo señalado en los párrafos precedentes, es que se plantea la necesidad de investigar la importancia del manejo de las habilidades emocionales y comunicativas en los docentes, ya que las emociones y las relaciones sociales están inmersas en los procesos pedagógicos que se desarrollan al interior de las instituciones educativas, y a partir del manejo y dominio emocional que el docente posea, se verá reflejado en la comunicación y relación docente-estudiante, aportando a un ambiente escolar favorable.

Asimismo, para aportara un clima escolar óptimo se deben transformar las prácticas docentes y las relaciones que establece el docente con sus estudiantes (MEN, 2015). En este sentido, la UNESCO (2013) menciona que "El clima de relaciones humanas que prevalece en las escuelas es una de las variables que más influye en el aprendizaje de los estudiantes en América Latina" (p. 5). Además, "Una relación fluida y de calidad alumno/profesor tendría una alta correlación con buenos resultados de los estudiantes" (p. 6). De igual forma, "El docente debe desarrollar habilidades de comunicación que le permitan efectuar contactos humanos eficaces y crear vínculos afectivos con el conocimiento, es decir, construir puentes entre los saberes y sus alumnos" (Osorio y Oviedo, 2009, p. 42). Según Motataianu (2014) "La forma en que un maestro se comunica con sus alumnos es importante, y si utiliza formas atractivas de mostrar información a través de la interacción, los resultados serán inmediatos" (p. 709). 
Igualmente, "La comunicación permite que los agentes de la interacción educativa: profesor y estudiantes, se relacionen mutuamente, generándose un proceso a través del cual uno de ellos contribuye a la formación del otro, en la medida en que esa relación les ayuda en su proceso de humanización (Osorio y Oviedo, 2009, p. 40).

Cabe destacar que la inteligencia emocional se ha venido investigando desde hace varios años, con teóricos como Mayer \& Salovey a partir de 1990, constituyéndose un campo de investigación interesante y pertinente a las exigencias y cambios del mundo actual. Así como también se han realizado diversas investigaciones en torno al ambiente del aula de clase destacando la importancia de este en el proceso de enseñanza y aprendizaje.

Debido a la importancia de este tema, este trabajo se propone investigar cuál es el nivel de dominio/manejo de las habilidades emocionales y comunicativas de los docentes de básica secundaria y media vocacional, a partir de los resultados del ISCE en las Pruebas Saber de $9^{\circ}$ grado de una institución educativa privada de la ciudad de Barranquilla en el componente de Ambiente Escolar al no alcanzar el puntaje máximo posible, y de esta forma generar una reflexión neuropedagógica que permita identificar la importancia de estos aspectos en el proceso de enseñanza y aprendizaje.

Es precisamente a través de la Neuropedagogía que se genera la reflexión; tomando como referente teórico la neurociencia cognitiva, basado en lo expuesto por Izaguirre (2017) "tiene injerencia en la educación, dado que es el soporte neuroanatómico y funcional de los procesos cognitivos que se dan en los mecanismos de enseñanza-aprendizaje, viabilizando la comprensión de los procesos cognitivos de la enseñanza-aprendizaje" (p. 87). Por su parte, la neurociencia cognitiva social concebida como "el estudio de los mecanismos neurobiológicos de los procesos superiores (teoría de la mente, empatía, autoconciencia, razonamiento moral, intencionalidad e imitación) que intervienen en la cognición social." (Grande, 2009, p. 2).

Como se menciona anteriormente la Cognición Social (CS) hace parte de la neurociencia cognitiva social:

Puede definirse como un proceso neurobiológico, psicológico y social, por medio del cual se perciben, reconocen y evalúan los eventos sociales, para construir una representación del ambiente de interacción de los individuos (Adolphs, citado por Uribe, 2010), y posteriormente generar el comportamiento social, es decir, la respuesta más adecuada según la circunstancia particular. Se relaciona con la percepción social (estadio inicial que evalúa las intenciones de los demás a través de su conducta -dirección de la mirada y movimiento corporal-) y el estilo atribucional (cómo se explica la conducta de otras personas) (Pelphry, Adolphs \& Morris, citado por Uribe, 2010, p. 2)

Por su parte, Zegarra (2014) afirma que "es un procesamiento cognitivo diferenciado para los estímulos sociales, que permite formar intenciones y atribuciones sobre uno mismo y los demás, articulando información para su uso en la interacción social ligada a un contexto" ( $p$. 28). 
Cabe destacar que existe una teoría que se relaciona con la Cognición Social: la Teoría de la Mente, la cual es definida como "La habilidad de entender el rol de otros individuos, así como de entender los puntos de vista o atribuir una intención a otro individuo" (...) también se define como "la capacidad de atribuir mente a otro sujeto distinto a uno mismo, y juega un rol fundamental en la cognición social" (Butman, 2001, p. 120).

Partiendo de lo anterior, las habilidades emocionales asociadas a la inteligencia emocional han sido investigadas por diversos autores desde hace muchos años y en dichas investigaciones se ha identificado la importancia de esta en los procesos de enseñanza y aprendizaje. Asimismo, se observa que los aspectos propios de la Cognición social y la Teoría de la Mente se relacionan con las habilidades emocionales y comunicativas del Modelo de Inteligencia Emocional de Mayer \& Salovey, junto con las Habilidades Sociales de Goldstein en las que se destacan la identificación, manejo y expresión de emociones, la toma de decisiones, las consecuencias de los actos, el procesamiento de la información no verbal y entender al otro.

\section{Método}

\section{Diseño}

La investigación se desarrolló bajo un enfoque de métodos mixtos, el cual plantea un problema que demanda el uso e integración de los enfoques cuantitativo y cualitativo (Hernández, Fernández, Baptista, 2014). Se eligió este enfoque ya que en la investigación se utilizaron técnicas de recolección de datos cuantitativas como es el caso del Test de Medición Estandarizado de la Inteligencia Emocional, el Cuestionario de Clima Motivacional de Clase y la Lista de Chequeo de Habilidades Sociales de Goldstein, así como también se utilizaron técnicas cualitativas como la revisión documental y el grupo focal. Todo ello con el fin de complementar los resultados de la investigación y de esta forma dar respuesta al problema planteado de forma completa.

La investigación desarrolló un Diseño Explicativo Secuencial (DEXPLIS) (Hernández, Fernández, Baptista, 2014) en el que se recolectaron y analizaron los datos del Test de Medición Estandarizado de la Inteligencia Emocional, el Cuestionario de Clima Motivacional de Clase y la Lista de Chequeo de Habilidades Sociales y, a partir de los resultados, se realizaron los grupos focales como medio para complementarlos y de esta forma obtener resultados e información más completa que permitió el análisis de los datos de forma complementaria y dar respuesta al planteamiento del problema.

\section{Participantes}

Con relación al establecimiento en el que se realizó la investigación es una institución educativa privada de la ciudad de Barranquilla, estrato seis, calendario B, bilingüe. Ofrece educación en los niveles de preescolar, básica primaria, básica secundaria y media vocacional. La muestra para el desarrollo y ejecución de la investigación se obtuvo por medio de un 
muestreo no probabilístico, los sujetos debían pertenecer a la institución educativa, ser docentes y estudiantes de secundaria y media vocacional, y, en el caso de los estudiantes, contar con la autorización de los padres de familia para participar.

Hicieron parte de la investigación 18 docentes de básica secundaria y media vocacional, de diversas áreas del conocimiento. Cabe destacar que las edades de los participantes oscilan entre los 23 y los 50 años, 10 son hombres y 8 son mujeres. En cuanto a los estudiantes participaron 131 estudiantes cuyas edades oscilan entre los 11 y 18 años pertenecientes a $6^{\circ}$, $7^{\circ}, 8^{\circ}, 9^{\circ}, 10^{\circ}$ y $11^{\circ}$ grado.

\section{Instrumentos y Procedimiento}

Para el desarrollo de la investigación se emplearon diversas técnicas e instrumentos que permitieron la recolección de la información. A continuación, se mencionan y se establece el orden en que se recolectaron los datos:

-Revisión documental a través de fichas técnicas, proceso en el cual se realizó la revisión teórica de las temáticas de la investigación.

-Cuestionario de Clima Motivacional de Clase (CMC-Q) (Alonso \& Fernández, 2008), este cuestionario fue respondido por los estudiantes.

-Lista de Chequeo de Habilidades Sociales de Goldstein (Rojas, 2017). La respondieron los docentes con el fin de identificar cómo son sus habilidades comunicativas en los procesos de interacción con sus estudiantes.

-MSCEIT Test de Inteligencia Emocional Mayer- Salovey-Caruso (Mayer, Salovey \& Caruso, 2016). A través de este test se identificó el estado de las habilidades de inteligencia emocional de los docentes.

-Grupos Focales con estudiantes y con docentes (por separado) a través de un protocolo de preguntas. Los grupos focales se desarrollaron con representantes de estudiantes de cada grado de la básica secundaria y media-vocacional con el fin de conocer la forma en que los estudiantes perciben a sus docentes en cuanto al manejo de las habilidades emocionales y habilidades comunicativas en sus clases y el ambiente escolar en general. Cabe destacar que con los estudiantes se realizó un grupo focal conformado por cada doce estudiantes pertenecientes a dos grados, es decir, 12 estudiantes de $6^{\circ}$ y $7^{\circ}$ grado, 12 estudiantes de $8^{\circ}$ y $9^{\circ}$ grado y 12 estudiantes de $10^{\circ}$ y $11^{\circ}$ grado. De igual forma, se desarrollaron grupos focales con los docentes a los que se les aplicó el Test de Inteligencia Emocional y Lista de Chequeo de Habilidades Sociales, con el fin de complementar y profundizar los datos recolectados; así se obtuvo información que enriqueció la respuesta al problema de investigación planteado, orientados a la percepción, valor y conocimiento del ambiente escolar por parte de los 
participantes (docentes y estudiantes). Es importante mencionar que se realizaron dos grupos focales con los docentes.

-Para el análisis de los datos cuantitativos se utilizó la estadística descriptiva a través de las medidas de tendencia central (media), medidas de variabilidad (desviación típica), coeficiente de correlación de Pearson.

-Para el análisis de los datos cualitativos se utilizó la triangulación de datos: Análisis categorial (Okuda\& Gómez, 2005), triangulación metodológica (Arias, 2009).

\section{Resultados}

\section{Percepción de los estudiantes frente a las habilidades de los docentes y el ambiente escolar}

Según lo manifestado por los estudiantes a través del CMC-Q, los alumnos valoraron a los docentes en puntuaciones que oscilaron entre 89 y 104 puntos, siendo la media de 94 puntos. De esta forma, 1 docente se ubicó en la media, representando un 6\%, 11 docentes se ubicaron por debajo de la media, lo cual representa un $61 \%, y, 6$ docentes se ubicaron por encima de la media representando un 32\%; con una desviación típica de 4 puntos, es decir, que en promedio los profesores se encuentran a 4 puntos con respecto a la media. Es importante mencionar que el máximo puntaje a obtener en este cuestionario era de 160 puntos y el mínimo de 32 puntos.

\section{Gráfico 1.}

Puntuaciones obtenidas por los docentes en el Cuestionario de Clima Motivacional de Clase (CMC-Q)

\section{Cuestionario Clima Motivacional de Clase (CMC-Q)}

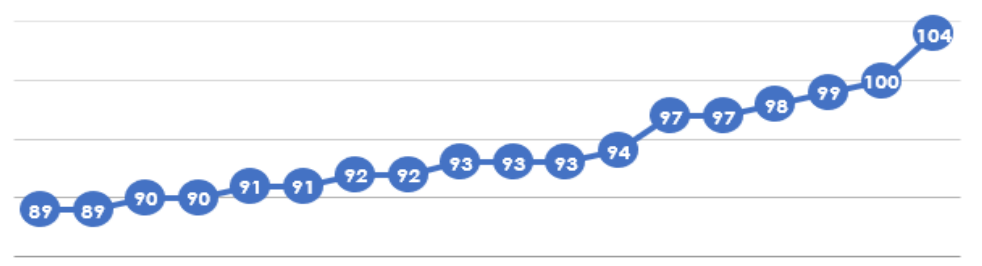

$\begin{array}{lllllllllllllllllll}\text { D9 } & \text { D10 } & \text { D14 } & \text { D15 } & \text { D5 } & \text { D16 } & \text { D6 } & \text { D8 } & \text { D1 } & \text { D4 } & \text { D18 } & \text { D12 } & \text { D7 } & \text { D11 } & \text { D3 } & \text { D2 } & \text { D17 } & \text { D13 }\end{array}$

Fuente: Elaboración propia

Por otra parte, en los grupos focales los estudiantes expresaron que algunos docentes son monótonos, no manifiestan emoción, sugieren que realicen actividades que impliquen la teoría y 
la práctica, que varíen sus estrategias didácticas. Mientras que hay otros docentes que muestran pasión por enseñar, les brindan seguridad e interés por participar en las clases. Aunque predominan los docentes que no los motivan y que carecen de actividades variadas y que despierten su interés por aprender. Por lo cual, es importante abordar los aspectos socioemocionales del proceso de aprendizaje. Cabe destacar que, el ambiente escolar por el nivel de desarrollo y nivel sociocultural no es una variable crítica, pero no está desarrollado como una competencia ocupacional de los docentes en pro de la calidad educativa, esto es coherente con lo señalado por los estudiantes. Es así como se identifica un perfil profesional y personal del docente que debe contribuir a un aprendizaje significativo, ya que los docentes no motivan al estudiante, no estimulan emocionalmente al aprendizaje, hay una ruptura entre el estímulo del docente y la emoción o motivación del estudiante.

\section{Percepción de los docentes frente a sus habilidades emocionales y comunicativas}

En cuanto a la aplicación del MSCEIT la mayor puntuación obtenida por los docentes fue de 131 puntos y la mínima de 77 puntos. La media se ubica en 104, estando 8 individuos por debajo de la media que representa un 44\%, 10 individuos se ubicaron por encima de la media que representa un 56\%, con una desviación típica de 12 puntos, es decir, que en promedio los profesores se encuentran a 12 puntos con respecto a la media. Cabe destacar, que, para que un docente sea experto debe obtener un puntaje mayor a 130, muy competente de 111 a 130 y competente emocionalmente debe ubicarse en puntuaciones de 90 a 110, puede mejorar si se ubica entre 70 y 89.

Gráfico 2.

Puntuaciones obtenidas por los docentes en el MSCEIT Test de Inteligencia Emocional

\section{MSCEIT Test de Inteligencia} Emocional

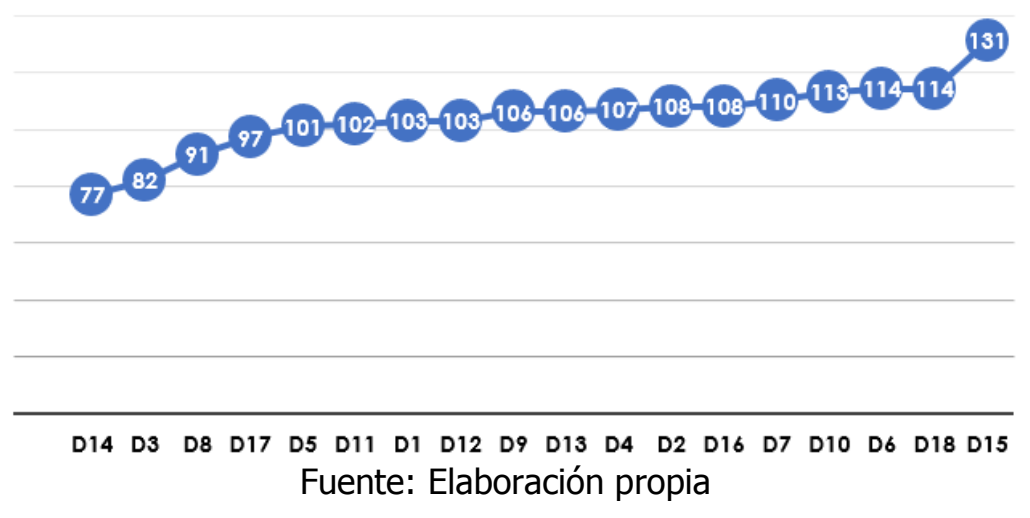

Con base en lo anterior, se puede señalar que los docentes no tienen conciencia de sus propias habilidades emocionales, son competentes porque son profesionales, por lo cual su 
destreza socioemocional debería estar equilibrada por su desempeño, ya que los docentes deben crear en el aula condiciones emocionales necesarias para el aprendizaje. El maestro que posea Inteligencia Emocional ayudará a construir aulas emocionalmente dispuestas para el aprendizaje significativo en sus alumnos. A partir de los resultados se infiere que son docentes típicos, a partir de su nivel de desarrollo profesional y por estar en una institución educativa de estrato socioeconómico seis, implicaría que la sociedad les ayuda a controlarse. Sin embargo, dos docentes deben mejorar de acuerdo con el test, pero todos los profesores deben mejorar su habilidad emocional para llegar a ser muy competentes o expertos emocionalmente, de esta forma favorecer las relaciones con los estudiantes y aportar al clima escolar.

En cuanto a la Lista de Chequeo de Habilidades Sociales de Goldstein la máxima puntuación es de 250 puntos y la mínima 50 puntos. La media se ubicó en 207 puntos con 9 individuos por debajo de la media, lo cual, representa un $50 \%$ y 9 docentes por encima de la media, representando un 50\%, con una desviación típica de 18 puntos, es decir, que en promedio los profesores se encuentran a 18 puntos con respecto a la media. Asimismo, teniendo en cuenta los resultados obtenidos en cada una de las habilidades de las cuales está compuesto este instrumento, es importante que los docentes mejoren, pues evidencian que tienen habilidades sociales de inhibición y otros son agresivos, por lo cual es necesario entrenarlos en la asertividad, que puedan manejar estas situaciones y sepan cómo gestionar socialmente situaciones de conflicto social. Cabe destacar que si los docentes no manejan sus emociones tendrán dificultades en la relación docente-estudiante.

\section{Gráfico 3.}

Puntuaciones obtenidas por los docentes en La Lista de Chequeo de Habilidades Sociales de Goldstein

\section{Lista de Chequeo Habilidades} Sociales Goldstein

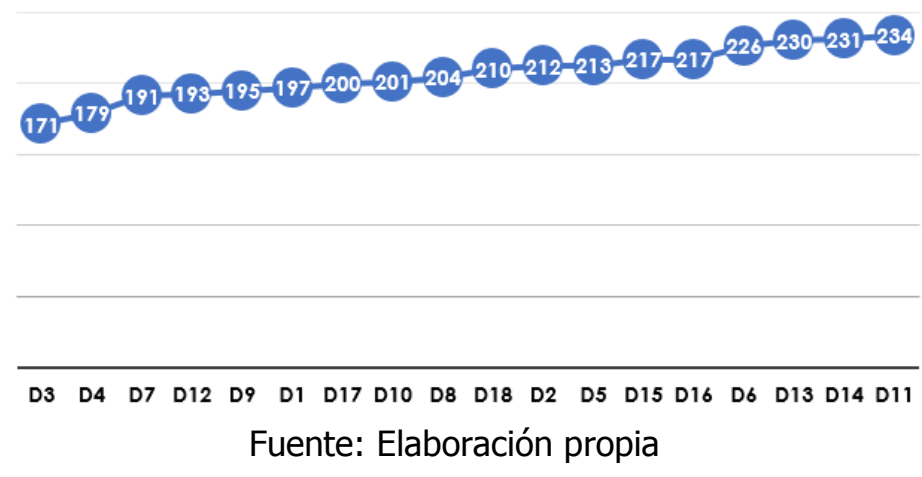

\section{Correlación de Variables (Coeficiente de Correlación de Pearson)}

A partir de la correlación entre el MSCEIT y las variables emocionales del CMC-Q, en ambos instrumentos los profesores obtuvieron puntuaciones variadas. Al realizar el proceso estadístico 
inferencial: coeficiente de correlación de Pearson se obtuvo $-0,19(-19 \%)$, por lo tanto, las variables son inversamente proporcionales y la correlación es muy débil, es decir: A mayor puntuación en CMC-Q, menor puntuación en MSCEIT. A menor puntuación en CMC-Q, mayor puntuación en MSCEIT.

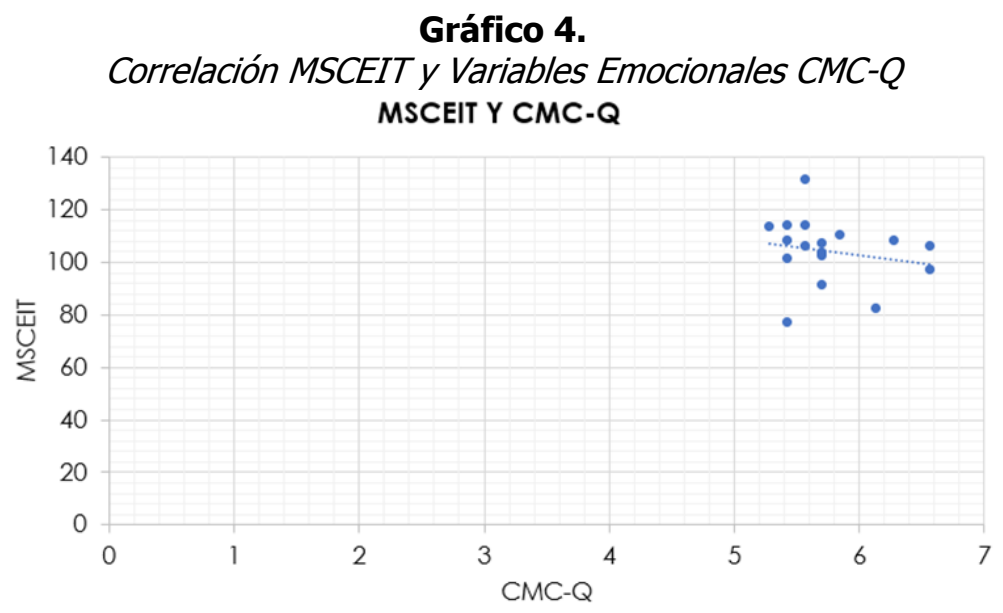

Fuente: Elaboración propia

En lo que respecta a la correlación entre la lista de chequeo de Goldstein y las variables comunicativas del CMC-Q se obtuvo una correlación de 0,16 (16\%), por lo tanto, las variables son directamente proporcionales y la correlación es muy débil, es decir: A mayor puntuación en CMC-Q, mayor puntuación en la Lista de Chequeo de Goldstein. A menor puntuación en CMC-Q, menor puntuación en la Lista de Chequeo de Goldstein.

Gráfico 5.

Correlación Lista de Chequeo Goldstein y Variables Comunicativas CMC-Q

Lista de Chequeo Goldstein, CMC-Q

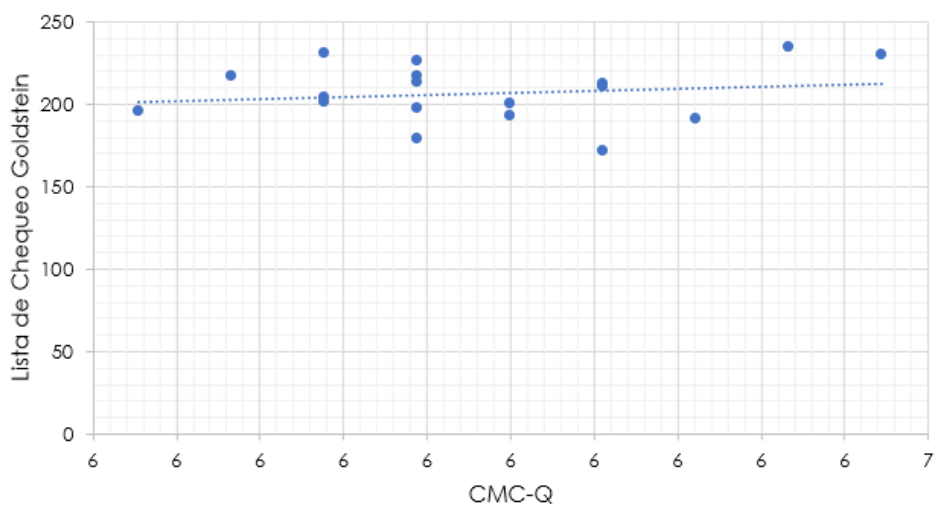

Fuente: Elaboración propia 
Teniendo en cuenta los resultados del coeficiente de Correlación de Pearson para las habilidades emocionales y comunicativas desde la perspectiva del docente en los instrumentos aplicados, la puntuación varió, puesto que, cada docente obtuvo una puntuación diversa, algunos poseen una puntuación alta y otros una puntuación baja, y, en el cuestionario de Clima Motivacional de Clase respondido por los estudiantes los resultados son homogéneos. Por ello, la correlación es baja, ya que la percepción del docente y estudiante difieren.

\section{Discusiones y conclusiones}

Teniendo en cuenta los resultados obtenidos, se evidencia que la percepción de los estudiantes y los docentes frente al ambiente escolar y las habilidades comunicativas y emocionales difieren entre sí, como manifestación negativa al ISCE en su componente de ambiente escolar.

Es evidente que no hay relación en la percepción del estudiante y la del docente debido a que el estudiante está percibiendo algo de lo que el docente no es consciente. Ante esto, se propone una reflexión sobre la relación docente-estudiante y las categorías mediadoras de la calidad y efectividad interaccional que contribuyan a un ambiente escolar pertinente a las metas educativas de la formación integral en el marco de las competencias para el siglo XXI, resaltando las habilidades comunicativas y emocionales, que subyacen a componentes neurobiológicos y socioemocionales de los participantes, es así, como una intervención desde estos componentes, favorece la relevancia de comprender la importancia de la Neuropedagogía en el proceso de enseñanza y aprendizaje. De acuerdo con García \& Valverde (2008) "La emoción es un factor clave en la conquista y construcción del aprendizaje. La implicación emocional del individuo se une a su cognición y habilidades formando un todo único que interviene en el proceso de enseñanza y aprendizaje, determinando en gran medida el éxito de este" (p. 82) por lo cual, es importante reconocer el valor de la emoción y motivación en los procesos de aprendizaje y enseñanza en las comunidades educativas, los diferentes resultados en la formación integral y mejorar la relación docente-estudiante para crear ambientes positivos y de calidad.

Se destaca que para logar la calidad educativa se debe tener en cuenta, entre sus dimensiones, la relación docente-estudiante como elemento predominante del ambiente escolar y profundizar en él desde el enfoque neuropedagógico para comprender la estructura neurobiológica del cerebro humano y su relación en los procesos motivacionales de enseñanza y aprendizaje, esto es respaldado por Izaguirre (2017) quien plantea que para lograr un ambiente de aprendizaje óptimo se deben tener en cuenta las motivaciones, la autoconfianza, aspectos emocionales de los estudiantes; puesto que hay conexiones neuronales importantes entre las áreas perceptivas y las áreas del cerebro que son claves para el proceso de aprendizaje escolar.

Además, es imperante resaltar la necesidad de que los maestros posean habilidades emocionales y comunicativas efectivas que le permitan generar ambientes propicios, altamente 
motivantes, respetuosos de las individualidades y necesidades de los participantes y sus propios procesos culturales en los procesos de enseñanza y aprendizaje. Por lo cual, las instituciones de formación profesional deben valorar e incluir elementos que vayan más allá de la instrucción disciplinar, otorgando valor a la formación en competencias del ser para el aprendizaje con más sentido y significado.

De igual forma, es importante la reflexión constante por parte de los docentes sobre su práctica pedagógica, a partir de lo expuesto por Ávila (2007) "la pedagogía siempre y necesariamente es una construcción discursiva que se da a partir de una reflexión consciente sobre, las prácticas, las políticas educativas, los procesos de enseñanza-aprendizaje (...)" ( $p$. 37). Teniendo en cuenta lo anterior, los docentes deben reflexionar desde las diferentes dimensiones y habilidades para analizar su quehacer pedagógico día a día y plantear acciones de mejora en las competencias tanto de lo personal, disciplinar y profesional, que contribuyan a favorecer procesos de enseñanza y aprendizaje, el ambiente escolar que apoyen a la búsqueda constante de la calidad educativa en las diferentes comunidades, para promover la enseñanza desde relaciones interpersonales empáticas, tolerantes y comprensivas de las aulas diversas, multiculturales y multiétnicas de la sociedad actual.

\section{Referencias}

Alonso, J., y Fernández, B. (2008). Development and initial validation of the Classroom motivational Climate Questionnaire (CMC-Q). Psicothema, 20(4), 883-889.

Arias, L. (2009). Interdisciplinariedad y Triangulación en Ciencias Sociales. Diálogos, Revista Electrónica de Historia, 10(1), 117-136.

Arias, I. (2018). Ambientes escolares: un espacio para el reconocimiento y respeto por la diversidad. Sophia, 14(2), 84-93. https://doi.org/10.18634/sophiaj.14v.2i.852

Aristulle, P., Paoloni, P. (2019). Habilidades socioemocionales en las comunidades educativas: aportes para la formación integral de los y las docentes. Revista Educación, 43(2). https://doi.org/10.15517/revedu.v43i2.28643

Ávila, R. (2007). Fundamentos pedagógicos hacia una comprensión del saber pedagógico. Cooperativa Editorial Magisterio.

Butman, J. (2001). La Cognición Social y la Corteza Cerebral. Revista Neurológica Argentina, (26), 117-122.

Cabello, R., Ruiz, D., y Fernández, P. (2010). Docentes emocionalmente inteligentes. Revista Electrónica Interuniversitaria de Formación del Profesorado, 13(1), 41-49. 
Cejudo, J., López, M. (2017). Importancia de la inteligencia emocional en la práctica docente: un estudio con maestros. Psicología Educativa, 23(1), 29-36. https://doi.org/10.1016/j.pse.2016.11.001

De Zubiría, J. (2018). ¿Cómo mejorar la calidad de la educación en Colombia? http://blogpedagogiadialogante.com/mejorar-la-calidad-la-educacion-colombia/

Esquivel, (2018). La inteligencia emocional para favorecer el clima y participación en el aula. Revista acta educativa, 6(15), 1-21.

Fernández, (2019). Educación: competencias, emociones y humor, perspectivas y estudios. Educación y Humanismo, 21(37), 51-66. http://doi.org/10.17081/eduhum.21.37.3365

García, N., y Valverde, J. (2008). Cerebro y emoción: reflexiones para contextos educativos. Revista Contextos de Educación, 20(25), 77-88.

Grande, I. (2009). Neurociencia social: El maridaje entre la psicología social y las neurociencias cognitivas. Revisión e introducción a una nueva disciplina. Anales de psicología, 25(1), 120.

Gutiérrez, A., y Buitrago, S. (2019). Las Habilidades Socioemocionales de los Docentes, herramientas de paz en la escuela. Praxis \& Saber, 10(24), 167-192. http://doi.org/10.19053/22160159.v10.n25.2019.9819

Hernández, R., Fernández, C., Baptista, P. (2014). Metodología de la Investigación. Mc Graw Hill.

Izaguirre, M. (2017). Neuroproceso de la enseñanza y del aprendizaje: metodología de la aplicación de la neurociencia en la educación. Alfaomegacolombiana S.A.

López, L., \& Oriol, X. (2016). The relationship between emotional competence, classroom climate and school achievement in high school students. Culture and Education, 28(1), 1- 26. http://doi.org/10.1080/11356405.2015.1120448

Mayer, J. D., \& Salovey, P. (1997). What is emotional intelligence:En P. Salovey y D. Sluyter (Eds.), Emotional Development and Emotional Intelligence: Implications for Educators (pp. 3-31). Basic Books.

Mayer, J., Salovey, P., \& Caruso, D. (2016). MSCEIT. Test de Inteligencia Emocional MayerSalovey-Caruso (N. Extremera, P., Fernández-Berrocal, adaptadores) ( ${ }^{\circ}$ ed.). TEA Ediciones.

Meiss, P. (2017). Educación de calidad: criterios de la UNESCO. https://www.emagister.com/blog/educacion-de-calidad-criterios-de-la-unesco/ 
Ministerio de Educación Nacional. (2011). Dirección de Calidad Viceministerio Preescolar Básica $y$ Media. https://www.mineducacion.gov.co/1759/articles285176_archivo_pdf_10_calidad.pdf

Ministerio de Educación nacional. (2014). Índice Sintético de la Calidad Educativa -ISCE. https://www.mineducacion.gov.co/1759/w3-article-397385.html?_noredirect=1

Ministerio de Educación Nacional (MEN). (2015). Ruta De Reflexión Y Mejoramiento Pedagógico "Siempre Dia E". Guía 4 Para Directivos Docentes El Ambiente Escolar Y El Mejoramiento De Los Aprendizajes. http://aprende.colombiaaprende.edu.co/sites/default/files/naspublic/Guia\%204_Ciclo\%2 03_V4ok.pdf

Ministerio de Educación Nacional (30 de marzo de 2016). Decreto 501 de 2016. Jornada Única en los establecimientos educativos oficiales y el Programa para la implementación de la Jornada Única y el Mejoramiento de la Calidad de la Educación Básica y Media. https://www.funcionpublica.gov.co/eva/gestornormativo/norma.php?i=69054

Monjas, M. (2013). Habilidades Sociales Y Comunicación Eficaz. En Sánchez, M., et. Al. Gestión positiva de conflictos y mediación en contextos educativos. Editorial Reus, S.A.

Motataianu, I. (2014). The empathy and communication - pride personality's dimensions of the teacher. Procedia - Social and Behavioral Sciences, 142(2014), 708-711. http://doi.org/10.1016/j.sbspro.2014.07.602

Morton, C. (2014). Exploring Teacher Emotional Intelligence And Its Impact On School Climate. [Disertación de Doctorado, Kansas State University].

Ortiz, A. (2015). Neuroeducación ¿cómo aprende el cerebro humano y cómo deberían enseñar los docentes? Ediciones de la U.

Okuda, M., Gómez, C. (2005). Métodos en investigación cualitativa: triangulación. Revista colombiana de psiquiatría, 34(1), 118-124.

Osorio, G., Oviedo, L. (2009). Inteligencia emocional y procesos pedagógicos. Editorial Universidad Nacional de Colombia.

Palomera, R., Fernández, P., y Brackett, M. (2008). La inteligencia emocional como una competencia básica en la formación inicial de los docentes: algunas evidencias. Revista Electrónica de Investigación Psicoeducativa, 6(15), 437-454.

Pertegal, M., Castejón, J., Martínez, M. (2011). Competencias socioemocionales en el desarrollo profesional del maestro. Educación XX1, 14(2), 237-260. 
Ramos, R., y López, M. (2020). Tensiones y desigualdades en las interacciones áulicas en estudiantes universitarios. Educación y Humanismo, 22(38). https://doi.org/10.17081/eduhum.22.38.3554

Reyes, M., Garrido, M. (2012). Relaciones interpersonales en la educación. Ediciones Pirámide.

Rojas, A. (2017). Lista de chequeo de habilidades sociales de Goldstein. https://kupdf. net/download/habilidades-sociales-goldsteincompleto_59794434dc0d60b423043370_pdf

Thorne, C., Centeno, M., Wetzell, M. (2009) El clima motivacional en la clase: evidencias empíricas en centros educativos En Herrera, D. (2009). Teorías contemporáneas de la motivación: una perspectiva aplicada. Fondo Editorial de la Pontificia Universidad Católica del Perú.

UNESCO. (2013). Análisis Del Clima Escolar: ¿Poderoso Factor que explica el Aprendizaje en América Latina y el Caribe? http://www.unesco.org/new/fileadmin/MULTIMEDIA/FIELD/Santiago/pdf/analisis-delclima-escolar.pdf

Uribe, C. (2010). Una breve introducción a la Cognición Social: procesos y estructuras relacionados.https://www.studocu.com/es/document/universidad-demanizales/procesos-psicologicos/otros/una-breve-introduccion-a-la-cognicion-socialprocesos-y-estructuras-relacionados/2550330/view

Vásquez, F. (2008). Inteligencia Emocional En Alumnos, Docentes Y Personal Administrativo De Una Universidad Privada De Barranquilla. Psicogente, 11(20), 164-181.

Zegarra, J. (2014). Neuropsicología de la cognición social. Breve revisión de los conceptos. Revista de Peruana de Psicología y Trabajo Social, 3(1), 27-36.

\section{Agradecimientos y ayuda financiera}

Agradecemos el financiamiento de la Universidad del Atlántico en el marco de la Primera convocatoria interna, que otorga apoyo económico para el desarrollo de trabajos de grado en investigación formativa - nivel pregrado y postgrado- 2018. 